\title{
Efficient generation of random multipartite entangled states using time-optimal unitary operations
}

\author{
A. Borras, ${ }^{*}$ A. P. Majtey, ${ }^{\dagger}$ and M. Casas ${ }^{*}$ \\ Departament de Física and IFISC-CSIC, Universitat de les Illes Balears, 07122 Palma de Mallorca, Spain
}

(Received 28 April 2008; published 19 August 2008)

\begin{abstract}
We review the generation of random pure states using a protocol of repeated two-qubit gates. We study the dependence of the convergence to states with Haar multipartite entanglement distribution. We investigate the optimal generation of such states in terms of the physical (real) time needed to apply the protocol, instead of the gate complexity point of view used in other works. This physical time can be obtained, for a given Hamiltonian, within the theoretical framework offered by the quantum brachistochrone formalism, the quantum analogue to the brachistochrone problem in classical mechanics [Carlini et al., Phys. Rev. Lett. 96, 060503 (2006)]. Using an anisotropic Heisenberg Hamiltonian as an example, we find that different optimal quantum gates arise according to the optimality point of view used in each case. We also study how the convergence to random entangled states depends on different entanglement measures.
\end{abstract}

DOI: 10.1103/PhysRevA.78.022328

PACS number(s): 03.67.Bg, 03.67.Ac

\section{INTRODUCTION}

One of the most fundamental concepts in the quantum description of nature is that of entanglement $[1,2]$. Entanglement constitutes a physical resource that lies at the heart of important information processes [3-5] such as quantum teleportation, superdense coding, and quantum computation. It has recently been proved that the generic entanglement, defined as the entanglement of random states, can be produced in a polynomial time using random two-qubit gates [6].

Random states and random unitary operators are the quantum analogs of random numbers and are two basic concepts in quantum information and quantum communication tasks. Random unitary operators are involved in the superdense coding of arbitrary states [7] while the classical capacity of a noisy quantum channel is saturated by random quantum states [8], just to mention two very significant applications. Both of them are well defined by the Haar measure which remains invariant under unitary transformation. Since the generation of random states is exponentially hard we study the production of states with the same distribution of entanglement, a task that can be performed with fewer physical resources [9].

Although a large amount of different multipartite entanglement measures has recently been proposed, a considerable amount of research has particularly been devoted to the study of multiqubit entanglement measures defined as the sum of bipartite entanglement measures over all (or an appropriate family of) the possible bipartitions of the full system [10-14]. There exist two popular entanglement measures for multiqubit pure states, one based on the von Neumann entropy of marginal density matrices and the other one based upon the linear entropy of those matrices. It has recently been shown that the von Neumann entropy based measure is able to grasp more features of highly entangled states than

\footnotetext{
*toni.borras@uib.es

†ana.majtey@uib.es

†montse.casas@uib.es
}

the linear entropy based measure [15]. It is then expected that when the efficient generation of random states is studied in the light of these entanglement measures, some differences will arise because, as a consequence of the "concentration of measure" phenomenon, these states are almost maximally entangled [16].

Emerson et al. [17] introduced a protocol for generating pseudorandom unitary operators. A circuit of repeated twoqubit gates was considered acting on a separable state of $N$ qubits. The unitary operation is given by the combination of two independent single-qubit rotations, chosen according to the invariant Haar measure at each time step, and a fixed two-qubit gate. The convergence to the Haar measure was shown to be polynomial with the number of qubits. Some experimental evidences have reinforced these results. A Markovian description of certain two-qubit gates has also been used to analytically prove that the convergence is reached in a polynomial time with the size of the system [9]. Recently, a numerical effort has allowed to identify the optimal twoqubit gate improving this analytical bound [18].

In these previous works convergence was studied in terms of gate complexity, i.e., the number of gates needed to reach the Haar distribution. In contraposition to the gate complexity, a new complexity concept for quantum algorithms has been proposed: The time complexity $[19,20]$, understood as the physical time needed to perform such algorithm. The minimization of this time is as important, from the experimental point of view, as the gate complexity. A considerable amount of work has recently been devoted to the timeoptimal quantum computation problem, with emphasis in the quantum brachistochrone formalism [21-24]. Making use of the analogy with the brachistochrone problem from classic mechanics, Carlini et al. [21] introduced a variational approach to obtain the optimal Hamiltonian and the optimal quantum evolution between initial and final given states. A geometric approach to solve this problem, based on the symmetry properties of the quantum states space, was addressed in [22]. The role of the entanglement within the quantum brachistochrone formalism was studied in [23].

A more general result has recently been formulated in terms of the variational principle to find the time-optimal 
duration of a unitary transformation [24]. This formulation is independent of the input state and because of that more general than the previously described one. The time-optimal way to obtain a two-qubit universal quantum gate was previously discussed using the Cartan decomposition scheme for unitary transformation and under the constraint that one-qubit gates can be performed arbitrarily fast $[25,26]$. To study the brachistochrone problem in relation with unitary operations, the quantum states space is replaced with the space of unitary operators. This formalism allows us to consider the constraint imposed by the finite amount of energy available in a physical experiment, as well as any other constraints imposed by experimental requirements or theoretical conditions. Then, the problem of finding the optimal parameters for the Hamiltonian, is reduced to the resolution of a set of ordinary differential equations [24].

The aim of this contribution is to study the possible differences between both formalisms i.e., the gate and time complexities. We also study the dependence of the convergence rate with different entanglement measures. The paper is organized as follows. In Sec. II we review the protocol to generate random pure states and discuss the dependence of the convergence time, in terms of the number of gates to be applied, with the entanglement measure. In Sec. III we study the physical time for the convergence. Finally, Sec. IV is devoted to summarize and discuss our results.

\section{GENERATION OF RANDOM BIPARTITE ENTANGLEMENT}

The efficient quantum circuit generating random quantum states of $N$ qubits is based on the iterative application of a two-qubit quantum gate $U_{i j}$ acting on qubits $i$ and $j$, arbitrarily drawn from the $N$-qubit system, at each time step. The quantum gate $U_{i j}$ is composed by the product of two singlequbit rotation gates $V_{i}$ and $V_{j}$ uniformly drawn from the Haar measure on $\mathrm{U}(2)$, and a fixed two qubit gate $W_{i j}$

$$
U_{i j}=V_{i} V_{j} W_{i, j}
$$

where $W_{i j}$ can be decomposed as

$$
W_{i j}=\left(v_{1} \otimes v_{2}\right) \exp \left(-\imath \sum_{k=x, y, z} \lambda_{k} \sigma_{k} \otimes \sigma_{k}\right)\left(u_{1} \otimes u_{2}\right),
$$

with the fixed rotations $v_{1,2}$ and $u_{1,2}$ acting only on one of the two qubits, and $\sigma_{k}$ are the Pauli matrices [27,28]. In our random entangling protocol the role of the fixed local rotations $u_{1,2}$ and $v_{1,2}$ should not be confused with the random local rotations $V_{i, j}$, which change at each step of the protocol. As we are just interested in the entanglement generation properties of the two-qubit gates we only need to consider the action of the nonlocal part of the decomposition (2), because its entangling power is the same when averaged over a large number of realizations [28,29]. The symmetries of such nonlocal action enables us to consider just a reduced range for the values of its parameters $\left(\lambda_{k} \in[0, \pi / 4], k=x, y, z\right)$. The qubits $i$ and $j$ upon which the gate $U_{i j}$ is applied can be chosen in several different ways, and each of them corresponds to different geometries of the system: Local and nonlocal [30]. In the nonlocal case qubits $i$ and $j$ are chosen randomly, the gate can act on two arbitrarily separated qubits. In the local case the gate can only act on two neighboring qubits. In this scheme we study both, periodic and open boundary conditions.

The results are qualitatively the same in the local and nonlocal case, so we only show those of the nonlocal couplings as representative of the typical behavior. The main difference is that a larger amount of two-qubit entangling gates are needed to converge to the entanglement of random states for the local geometry.

Our goal is to reproduce the entanglement of typical random states, to such an end we averaged a large enough number of realizations (typically $10^{3}$ ) in order to have small statistical fluctuations.

The genuine multipartite entanglement $E$ of a $N$-qubit state can be expressed as

$$
\begin{aligned}
E & =\frac{1}{(N / 2)} \sum_{m=1}^{(N / 2)} E^{(m)}, \\
E^{(m)} & =\frac{1}{N_{\text {bipart }}^{m}} \sum_{i=1}^{N_{\text {bipart }}^{m}} E(i) .
\end{aligned}
$$

Here, $E(i)$ stands for the entanglement associated with one, single bipartition of the $N$-qubits system. The quantity $E^{(m)}$ gives the average entanglement between subsets of $m$ qubits and the remaining $N-m$ qubits constituting the system. The average is performed over the $N_{\text {bipart }}^{(m)}$ nonequivalent ways to do such bipartitions, which are given by

$$
\begin{gathered}
N_{\text {bipart }}^{m}=\left(\begin{array}{c}
N \\
m
\end{array}\right) \quad \text { if } m \neq N / 2, \\
N_{\text {bipart }}^{N / 2}=\frac{1}{2}\left(\begin{array}{c}
N \\
N / 2
\end{array}\right) \quad \text { if } m=N / 2 .
\end{gathered}
$$

Different $E^{(m)}$ represent different entanglement properties of the state, this is why all these entanglement measures must be computed to capture all the entanglement properties of the state. The global multiqubit entanglement is given by the average of the $(N / 2)$ different $E^{(m)}$ for any state $|\Psi\rangle$.

We use two types of entanglement measures, $E_{L}$ and $E_{v N}$, respectively, based on two different measures for the mixedness of the marginal density matrices $\rho_{i}$ associated with the bipartitions:

(i) the linear entropy $S_{L}=\frac{2^{m}}{2^{m}-1}\left[1-\operatorname{Tr}\left(\rho_{i}^{2}\right)\right]$,

(ii) the von Neumann entropy $S_{v N}=-\frac{1}{m} \operatorname{Tr}\left(\rho_{i} \log _{2} \rho_{i}\right)$.

If one uses the linear entropy $S_{L}, E_{L}^{(1)}$ turns out to be the well-known Meyer-Wallach multipartite entanglement measure [31] that Brennen showed to coincide with the average of all the single-qubit linear entropies [32]. This measure was later generalized by Scott to the case in which all possible bipartitions of the system were considered [33].

We study the convergence according to different measures of multiqubit entanglement based upon bipartitions. We characterized the global entanglement with the average of the bipartite entanglement measures associated with the $2^{N}-1$ bipartitions of the $N$-qubit system. 
We compare the evolution towards the convergence of the global entanglement with the evolution of the entanglement of the most balanced bipartition $E^{(N / 2)}$ and the entanglement of the most unbalanced one $E^{(1)}$. We introduce the auxiliary normalized quantity

$$
\Delta E=\frac{E_{\text {Haar }}-\langle E\rangle}{E_{\text {Haar }}},
$$

which decays exponentially with the number of iterations. $\Delta E$ will make easy the comparison between different bipartitions. The saturation value $E_{\mathrm{Haar}}$, is the mean value of the entanglement of the Haar distribution given in [16,34] and $\langle E\rangle$ is the averaged entanglement over system realizations. We choose the initial state to be the separable state $|00 \cdots 0\rangle$ without losing generality. The optimal time is defined as the number of gates required to reach $\Delta E=0.01$. We fixed the two-qubit gate $\vec{\lambda}=(\pi / 4,0,0)$ which is known to be an optimal gate in the nonlocal couplings case [18]. The results are qualitatively the same for any other choice of the gate.

Figure 1 shows that the convergence rates do not depend on the dimension of the chosen bipartition if the entanglement is quantified with the linear entropy. However, there exist small differences between the convergence rates if we use the von Neumann entropy as the measure of entanglement. We have a little faster convergence for the most unbalanced bipartition. This behavior is more visible for higher dimensional states, the larger the number of qubits, the larger the difference between the convergence rates. These results imply that when working with the linear entropy, it is enough to consider the convergence of the Meyer-Wallach entanglement measure. If the von Neumann entanglement measure is used, one should consider the convergence of the entanglement of the most balanced bipartitions $E^{(N / 2)}$, because it is the one with a longer convergence time.

\section{GATE COMPLEXITY VS TIME COMPLEXITY}

In this section we are interested in the relation between the number of gates in the circuit in order to reach the Haar distribution and the physical time to perform this operation. As an example we choose the Heisenberg Hamiltonian

$$
H=-\sum_{j} J_{j} \sigma_{j}^{1} \sigma_{j}^{2}+\sum_{a} B^{a} \sigma_{z}^{a}
$$

where $J_{j}$ are anisotropic couplings $(j=x, y, z), B^{a}(t)$ $(a=1,2)$ is an external magnetic field in the $z$ direction, and $\sigma_{j}^{1}=\sigma_{j} \otimes I, \sigma_{j}^{2}=I \otimes \sigma_{j}$, with $\sigma_{j}$, as before, the Pauli matrices. The local magnetic terms appearing in (8) are needed to perform the two-qubit gate operation and are not related to the random single-qubit rotations $V_{i, j}$ introduced in Sec. II.

The optimal unitary evolution operator for the Heisenberg Hamiltonian (8) was obtained in [24]. Using the results of the quantum brachistochrone formalism the optimal time for some particular gates was calculated.

We focus our efforts in the optimal entangler gate $U_{\phi}$,
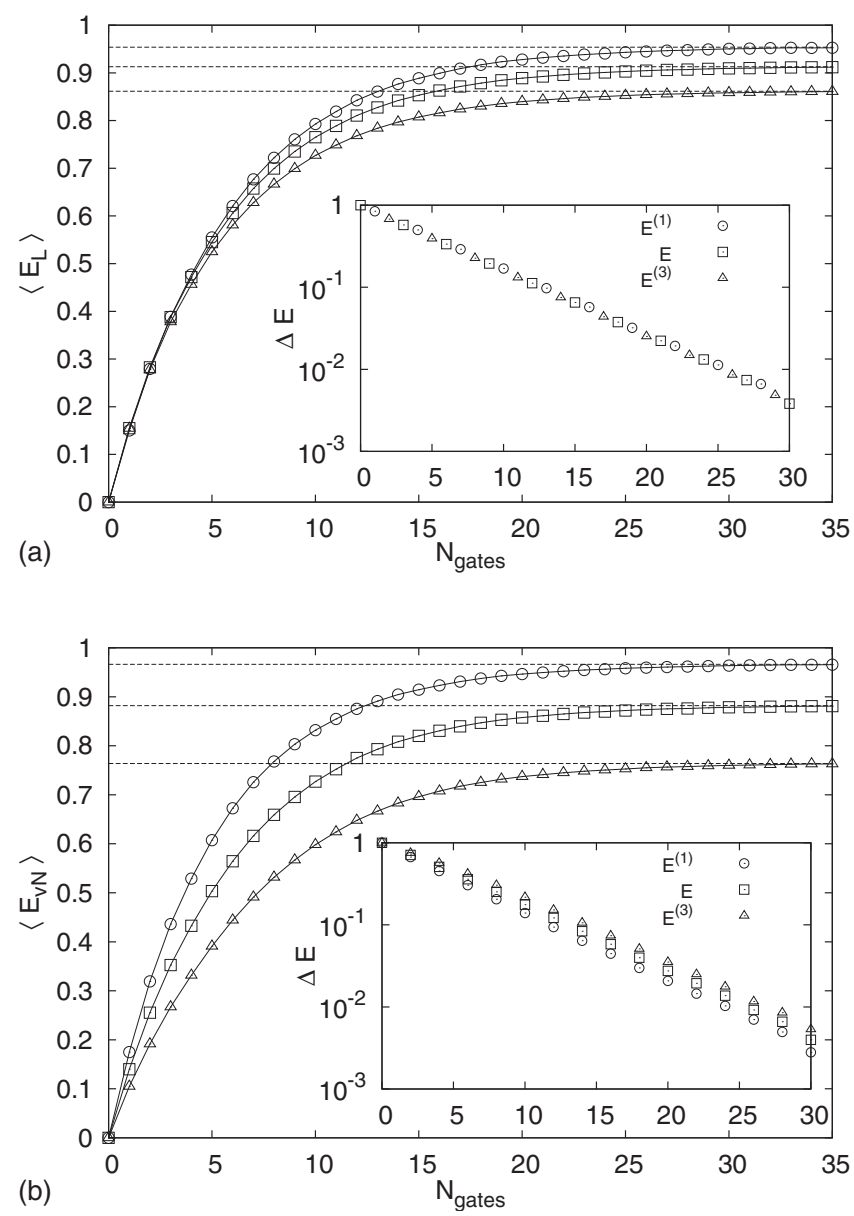

FIG. 1. Typical numerical simulation using the random circuit in a system of six-qubit. The entanglement average of the Haar measure, represented by dashed lines is reached in $N_{\text {gates }}$ steps. Top linear entropy as a measure of entanglement, bottom von Neumann entropy. We plot the global entanglement (squares) and the entanglement for the most balanced (triangles) and most unbalanced (circles) bipartitions of the system. Inset: Decay rates for the different entanglement measures, the rate is the same for the linear entropy and little differences are observed for the von Neumann entropy for different bipartitions of the system.

$$
U_{\phi}:=\left(\begin{array}{cccc}
\cos \phi & 0 & 0 & \sin \phi \\
0 & 1 & 0 & 0 \\
0 & 0 & 1 & 0 \\
-\sin \phi & 0 & 0 & \cos \phi
\end{array}\right),
$$

with the angle $\phi \in[0, \pi]$. This gate applied to an initial separable state produces a $\phi$-dependent entangled state. When this gate is applied to the separable state $|00\rangle$ it produces a maximally entangled state. The optimal time duration to implement the entangler gate assuming the finite energy condition is given by $\omega t_{\phi}=\pi \sqrt{x(1-x / 2)}$, where $x=\phi / \pi$ and $\omega$ is a constant given by the constraint. Since the only effect of the external parameter $\omega$ is to rescale the time, in the following we take $\omega=1$ as this choice will not modify our results. We study the convergence rate to the Haar distribution of this gate in terms of the physical time. This physical time is 


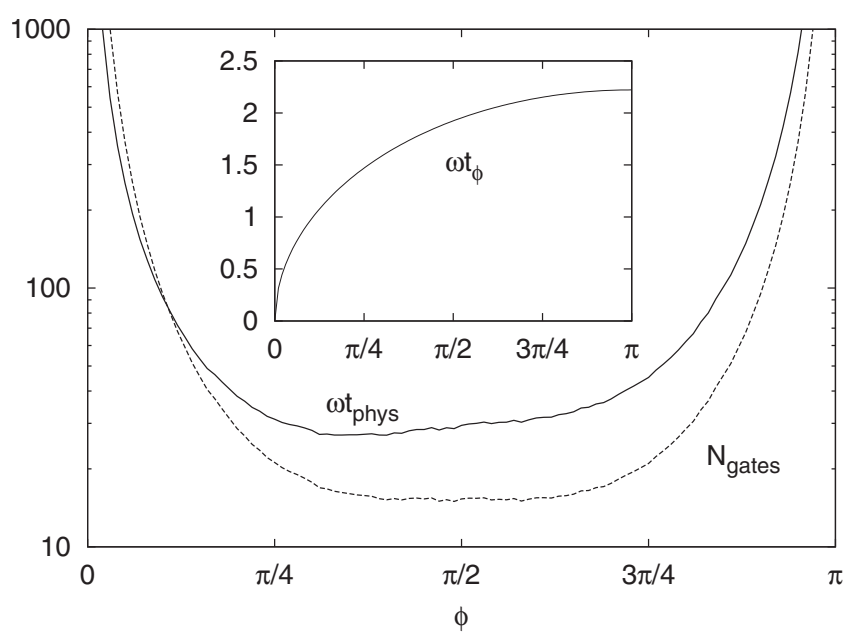

FIG. 2. Number of gates (dashed line) and physical time (solid line) to reach the convergence to the mean value of the Haar entanglement as a function of the gate parameter $\phi$ for a system of four qubits. Inset: Optimal time to implement the entangler $U_{\phi}$ gate as a function of $\phi$. As we have chosen units in which Planck's constant $\hbar$ is equal to 1 , all depicted quantities are dimensionless.

obtained as the product of the total number of gates $N_{\text {gates }}$ times the optimal time $t_{\phi}\left(t_{\text {phys }}=N_{\text {gates }} t_{\phi}\right)$. As in this case the final result is not affected by the choice of the measure of entanglement, we determine the convergence using $E_{L}$ as a measure for the mixedness of the marginal density matrices. As before we consider that the convergence is reached for $\Delta E=0.01$.

Figure 2 shows that a gate as simple as $U_{\phi}$, which depends just on the parameter $\phi$, is enough to reveal the differences between the gate-complexity and time-complexity concepts. The curve $N_{\text {gates }}$ can be viewed as the time that would be necessary to reach the convergence if all the gates took the same time $t_{\phi}=1$ to perform each evolution. In such case, all gates with values of $\phi$ in the interval $(\pi / 4,3 \pi / 4)$ would contribute to a reasonably efficient algorithm. The optimal gate would be attained by $\phi=\pi / 2$. But, as can be seen in the inset of Fig. 2, the optimal time $t_{\phi}$ needed to perform each iteration is far from being the same for all the values of $\phi$. It increases with the value of $\phi$, and it is negligible for values of $\phi$ near zero, because the entangling gate becomes the identity at $\phi=0$. If we combine both magnitudes, we obtain the total physical time $t_{\text {phys }}$, which inherits the main properties of $N_{\text {gates }}$ and $t_{\phi}$. The global behavior resembles that of $N_{\text {gates }}$, both of them diverge for the extremal values $\phi=0$ and $\phi=\pi$ while attaining their lower and optimum values for central values of $\phi$. The asymmetry of $t_{\text {phys }}$ compared to $N_{\text {gates }}$ comes from the behavior of $t_{\phi}$. While $N_{\text {gates }}$ is symmetric with respect to its optimum and central value $\phi$ $=\pi / 2$, the optimum gate according to $t_{\text {phys }}$ corresponds to a lower value of such parameter $(\phi \approx \pi / 3)$.

The case studied in this section is a good example of the role that the time complexity can play when designing an efficient quantum algorithm. According to the gate complexity there exist a large range of values of $\phi$ for which the random states generating algorithm is quite efficient. If one introduces the time complexity argument this degeneracy is broken. The difference between the time needed by these efficient gates is not huge but it is enough to be taken into account, especially in a situation where the algorithm must be run for a large number of times.

\section{SUMMARY AND DISCUSSION}

We have studied the generation of multipartite entangled states considering a protocol of a two-qubit fixed gate combined with two one-qubit random rotations. A comparison was made between the gate complexity and the time complexity revealing that they are two different optimality problems. In a real case both complexities should be taken into account, and the optimal gate would be one with a reasonable good behavior according to both points of view. Nevertheless, when the algorithm must be run a large number of times, the optimization of time complexity is mandatory. In the example studied in Sec. III, the time complexity optimization allowed us to find an optimal gate between the whole family of gates which are almost equally efficient according to the gate complexity.

The quantum brachistochrone formalism seems to be a promising approach for the treatment of the time complexity problem. Following the work of Carlini et al. [24] it is possible to obtain the optimal way to realize a given quantum gate, introducing in this derivation any constraint given by the experimental setup.

We also focused on the possible dependence of the resulting entanglement on the different allowed bipartitions of the system. We found that when the linear entropy is considered, the convergence rates are independent of the bipartition scheme. However, the convergence rates are different if we use the von Neumann entropy. In this case the most balanced bipartition should be considered to guarantee the convergence. These results are independent of the possible, local or nonlocal, geometries of the system.

\section{ACKNOWLEDGMENTS}

This work was partially supported by the MEC Grant No. FIS2005-02796 (Spain). A.B. acknowledges support from MEC through FPU Grant No. AP-2004-2962 and A.P.M. acknowledges support of MEC Contract No. SB-2006-0165.
[1] I. Bengtsson and K. Zyczkowski, Geometry of Quantum States: An Introduction to Quantum Entanglement (Cambridge University Press, Cambridge, 2006).

[2] M. B. Plenio and S. Virmani, Quantum Inf. Comput. 7, 1
(2007).

[3] M. A. Nielsen and I. L. Chuang, Quantum Computation and Quantum Information (Cambridge University Press, Cambridge, 2000) 
[4] Introduction to Quantum Computation and Information, edited by H.-K. Lo, S. Popescu, and T. Spiller (World Scientific, River Edge, 1998).

[5] The Physics of Quantum Information, edited by D. Bouwmeester, A. Ekert, and A. Zeilinger (Springer, Berlin, 2000).

[6] O. C. O. Dahlsten, R. Oliveira, and M. B. Plenio, J. Phys. A 40, 8081 (2007).

[7] A. Harrow, P. Hayden, and D. Leung, Phys. Rev. Lett. 92, 187901 (2004).

[8] S. Lloyd, Phys. Rev. A 55, 1613 (1997).

[9] R. Oliveira, O. C. O. Dahlsten, and M. B. Plenio, Phys. Rev. Lett. 98, 130502 (2007).

[10] Y. S. Weinstein and C. S. Hellberg, Phys. Rev. Lett. 95, 030501 (2005).

[11] A. R. R. Carvalho, F. Mintert, and A. Buchleitner, Phys. Rev. Lett. 93, 230501 (2004).

[12] L. Aolita and F. Mintert, Phys. Rev. Lett. 97, 050501 (2006).

[13] J. Calsamiglia, L. Hartmann, W. Dür, and H. J. Briegel, Phys. Rev. Lett. 95, 180502 (2005).

[14] A. Borras, AR Plastino, J. Batle, C. Zander, M. Casas, and A. Plastino, J. Phys. A 40, 13407 (2007).

[15] A. Borras, M. Casas, A. R. Plastino, and A. Plastino, Int. J. Quantum Inf. 6, 605 (2008).

[16] P. Hayden, D. W. Leung, and A. Winter, Commun. Math. Phys. 265, 95 (2006).

[17] J. Emerson, Y. S. Weinstein, M. Saraceno, S. Lloyd, and D. G. Cory, Science 302, 2098 (2003).
[18] M. Žnidarič, Phys. Rev. A 76, 012318 (2007).

[19] T. Schulte-Herbrüggen, A. Spörl, N. Khaneja, and S. J. Glaser, Phys. Rev. A 72, 042331 (2005).

[20] P. Wocjan, D. Janzing, and T. Beth, Quantum Inf. Comput. 2, 117 (2002).

[21] A. Carlini, A. Hosoya, T. Koike, and Y. Okudaira, Phys. Rev. Lett. 96, 060503 (2006).

[22] D. C. Brody and D. W. Hook, J. Phys. A 29, L167 (2006).

[23] A. Borras, C. Zander, A. R. Plastino, M. Casas, and A. Platino, Europhys. Lett. 81, 30007 (2008).

[24] A. Carlini, A. Hosoya, T. Koike, and Y. Okudaira, Phys. Rev. A 75, 042308 (2007).

[25] N. Khaneja and S. J. Glaser, Chem. Phys. 267, 11 (2001).

[26] J. Zhang, J. Vala, S. Sastry, and K. B. Whaley, Phys. Rev. A 67, 042313 (2003).

[27] N. Khaneja, R. Brockett, and S. J. Glaser, Phys. Rev. A 63, 032308 (2001).

[28] B. Kraus and J. I. Cirac, Phys. Rev. A 63, 062309 (2001).

[29] J. Batle, M. Casas, A. R. Plastino, and A. Plastino, Opt. Spectrosc. 99, 371 (2005).

[30] Y. Most, Y. Shimoni, and O. Biham, Phys. Rev. A 76, 022328 (2007).

[31] D. A. Meyer and N. R. Wallach, J. Math. Phys. 43, 4273 (2002).

[32] G. K. Brennen, Quantum Inf. Comput. 3, 619 (2003).

[33] A. J. Scott, Phys. Rev. A 69, 052330 (2004).

[34] E. Lubkin, J. Math. Phys. 19, 1028 (1978). 